\title{
Diagnóstico das condições de coleta e transporte externos de resíduos biológicos em unidades hospitalares brasileiras
}

\author{
Diagnosis of conditions for the collection and external transport of biological waste in Brazilian \\ hospital units
}

Diagnóstico de las condiciones externas de recogida y transporte de residuos biológicos en unidades hospitalarias brasileñas

Recebido: 23/11/2021 | Revisado: 03/12/2021 | Aceito: 06/12/2021 | Publicado: 16/12/2021

\author{
Ingrid Felizardo Chaves Cicca \\ ORCID: https://orcid.org/0000-0002-1674-494X \\ Universidade Federal Rural do Rio de Janeiro, Brasil \\ E-mail: ingrid.cicca@gmail.com \\ Fabiola de Sampaio Rodrigues Grazinoli Garrido \\ ORCID: https://orcid.org/0000-0001-5177-1241 \\ Universidade Federal Rural do Rio de Janeiro, Brasil \\ E-mail: fabiola_srg@yahoo.com.br
}

\begin{abstract}
Resumo
Em pleno século XXI, os lixões verdadeiros depósitos de lixo a céu aberto, ainda prevalecem como cenário atual e dominante no Brasil. Apesar da Política Nacional de Resíduos Sólidos (Lei 12.305/10), em vigor desde 2010, ter estabelecido o fim destes locais, sua implantação ainda não foi efetivada. Frente a ausência de alternativas ambientalmente adequadas para a disposição final de resíduos, inclusive da área de saúde, em muitos municípios brasileiros, segundo dados de 2020 da Associação Brasileira de Empresas de Limpeza Urbana, não há outra opção senão dispô-los em lixões. Nesse contexto, a presente pesquisa buscou compreender, por meio de entrevistas semiestruturadas com gestores que trabalham em hospitais públicos e privados do Brasil como é feito o acompanhamento e monitoramento interno e externo dos resíduos gerados. A metodologia contemplou, ainda, consulta a trabalhos científicos e dados estatísticos abertos junto a órgãos públicos. Considerou-se a responsabilidade compartilhada entre o gerador e os prestadores de serviços contratados pelas instituições, bem como a complexidade das atividades externas frente a uma série de variáveis de riscos, entre eles de acidentes, riscos físicos, riscos biológicos e riscos ocupacionais para os trabalhadores. Concluiu-se que nas etapas internas, os hospitais, de maneira geral atendem as exigências dos órgãos reguladores. No entanto, nas etapas externas de gerenciamento de resíduos constatou-se baixa frequência de auditorias ambientais externas, impossibilitando avaliações periódicas quanto o grau de implementação e a eficiência dos planos e programas no controle da poluição ambiental e oportunidades de melhorias relacionadas aos processos de coleta, evitando não-conformidades facilmente identificáveis em vistorias.

Palavras-chave: Resíduos biológicos; Coleta externa; RSS; Transporte de resíduos de saúde.
\end{abstract}

\begin{abstract}
In the middle of the 21st century, open-air dumps still prevail as the current and dominant scenario in Brazil. Although the National Solid Waste Policy (Law 12,305/10), in force since 2010, has established the end of these sites, its implementation has not yet been carried out. Faced with the absence of environmentally adequate alternatives for the final disposal of waste, including from the health area, in many Brazilian municipalities, according to data from the Brazilian Association of Urban Cleaning Companies, there is no other option but to dispose of them in dumps. In this context, this research sought to understand, through interviews with managers who work in public and private hospitals in Brazil, how the internal and external monitoring and monitoring of the generated waste is carried out. The methodology also included consultation of scientific works and statistical data opened with public bodies. The shared responsibility between the generator and the service providers hired by the institutions was considered, as well as the complexity of external activities against a series of risk variables, including accidents, physical risks, biological risks, and occupational risks for workers. It was concluded that in the internal stages, hospitals, in general, meet the requirements of regulatory bodies. However, in the external stages of waste management, a low frequency of external environmental audits was found, making it impossible to periodically assess the degree of implementation and the efficiency of plans and programs in the control of environmental pollution and opportunities for improvement related to the collection processes, avoiding easily identifiable non-conformities in inspections.
\end{abstract}

Key words: Biological waste; External collection; RSS; Health waste transport. 


\section{Resumen}

A mediados del siglo XXI, los vertederos, verdaderos vertederos de basura al aire libre, aún prevalecen como el escenario actual y dominante en Brasil. Si bien la Política Nacional de Residuos Sólidos (Ley 12.305 / 10), vigente desde 2010, ha establecido el final de estos sitios, aún no se ha llevado a cabo su implementación. Ante la ausencia de alternativas ambientalmente adecuadas para la disposición final de residuos, incluso del área de salud, en muchos municipios brasileños, según datos de la Asociación Brasileña de Empresas de Limpieza Urbana, no queda otra opción que eliminarlos en botaderos. En este contexto, esta investigación buscó comprender, a través de entrevistas con gerentes que laboran en hospitales públicos y privados en Brasil, cómo se realiza el monitoreo y monitoreo interno y externo de los residuos generados. La metodología también incluyó la consulta de trabajos científicos y datos estadísticos abiertos con organismos públicos. Se consideró la corresponsabilidad entre el generador y los prestadores de servicios contratados por las instituciones, así como la complejidad de las actividades externas frente a una serie de variables de riesgo, que incluyen accidentes, riesgos físicos, riesgos biológicos y riesgos laborales para los trabajadores. Se concluyó que, en las etapas internas, los hospitales, en general, cumplen con los requisitos de los organismos reguladores. Sin embargo, en las etapas externas de la gestión de residuos, se encontró una baja frecuencia de auditorías ambientales externas, lo que imposibilitó evaluar periódicamente el grado de implementación y la eficiencia de los planes y programas en el control de la contaminación ambiental y las oportunidades de mejora relacionadas con los procesos de cobranza, evitando no conformidades fácilmente identificables en las inspecciones.

Palabras clave: Residuos biológicos; Colección externa; RSS; Transporte de residuos sanitarios.

\section{Introdução}

A coleta e o transporte externos de resíduos de serviços de saúde (RSS) caracterizam-se por atividades que representam riscos ao trabalhador, à saúde pública e ao meio ambiente. Essas atividades se tornam ainda mais suscetíveis à riscos quando não são respeitadas as determinações estabelecidas pelos órgãos competentes, em especial da Agência Nacional de Vigilância Sanitária (ANVISA) e o Conselho Nacional de Meio Ambiente (CONAMA). O gerenciamento de resíduos de serviços de saúde está previsto nas resoluções da ANVISA (RDC 222/2018) e CONAMA 358/2005, no qual estão estabelecidas as diretrizes gerais para o tema. Na RDC 222/2018 constam os critérios para a elaboração do Plano de gerenciamento de resíduos (PGRSS), englobando os processos de segregação, acondicionamento, armazenamento, transporte, tratamento e disposição final, além de aspectos organizacionais e de recursos humanos. Já na resolução CONAMA 358/2005 a abordagem é acerca da preservação dos recursos naturais e do meio ambiente. Para tal, estabelece aos órgãos ambientais estaduais e municipais a responsabilidade perante a definição dos critérios para determinação dos tipos serviços passíveis licenciamento ambiental e obrigatoriedade da apresentação do PGRSS como parte integrante da conformidade ambiental.

A quantidade de resíduos gerados anualmente nos estabelecimentos de saúde é muito significativa. Segundo Alves et al. (2012) o crescimento na geração de resíduos de serviços de saúde se dá em virtude dos avanços tecnológicos e, consequentemente, na modernização do atendimento hospitalar. Para Sanches (1995) o aumento considerável dos resíduos de serviços de saúde ocorre pelo uso crescente de materiais descartáveis e a complexidade da atenção à saúde. Para Faria (2007) a produção de bens de consumo de uso único está relacionada diretamente com a quantidade e qualidade dos resíduos gerados. A ausência de ações efetivas que garantam um sistema seguro e adequado do gerenciamento de resíduos em todas suas etapas pode contribuir para o agravamento dos desafios existentes no cenário de resíduos no Brasil, que não são poucos. A Política Nacional de Resíduos Sólidos (Lei no . 12.305/2010), aprovada em 2010, previa a proibição dos lixões em todo o país em 2014. No entanto, os números da ABRELPE, demonstrados no relatório Panorama de Resíduos Sólidos no Brasil 2020, indicam que, em 2019, das 253 mil toneladas de resíduos de serviços de saúde (RSS) coletados, o equivalente 36,2\% tiveram como destinação os lixões.

Dado o potencial de riscos de acidentes de trabalho e impacto ambiental vinculados às atividades de transporte externo de resíduos perigosos - em especial os resíduos classificados como biológicos - buscou-se com esta pesquisa diagnosticar junto as unidades hospitalares, públicas e privadas, em diversas regiões do Brasil, o atual cenário das condições de coleta e transporte externos deste grupo de resíduos, bem como mapear a percepção dos gestores responsáveis pelas atividades 
de gerenciamento de RSS quanto a qualidade dos serviços prestados e de que maneira monitoram a conformidade legal das empresas contratadas.

\section{Metodologia}

\subsection{Definição da Amostra e da Estratégia de Execução da Pesquisa}

A primeira etapa da metodologia aplicada consistiu no mapeamento dos hospitais de grande porte no Brasil, tendo em vista que são os que concentram o maior número de leitos e consequentemente demandam mais pelos serviços de coleta e transporte externo de resíduos. As principais referências utilizadas neste mapeamento foram: a publicação Cenário dos Hospitais no Brasil 2019; consulta em base de dados do Cadastro Nacional de Estabelecimentos de Saúde (CNES); e a lista dos melhores hospitais, de acordo com o World's Best Hospital 2020. Na definição da amostra foi levada em consideração a proporção da participação das regiões e dos estados no cenário brasileiro de saúde.

O público-alvo da pesquisa consistiu nos profissionais que atuam, em hospitais públicos e privados no Brasil, como responsáveis diretamente pelo gerenciamento de resíduos de serviços de saúde. Foram realizadas análises qualitativas sobre as condições de coleta e transporte externos de resíduos biológicos constantes em publicações científicas, consulta em bases de dados abertas, legislações e normas técnicas e regras de licenciamento ambiental para transporte de resíduos perigosos.

Após a aprovação junto ao Comitê de Ética em Pesquisa (CEP), os dados foram coletados por meio de questionário online, com a devida disponibilização do Termo de Consentimento Livre e Esclarecido (TCLE). Na análise dos resultados, levou-se em consideração o caminho metodológico apontado por Fachin (2001) e Rey (2005). Considerou-se como método comparativo a definição de Fachin (2001, p.117):

... consiste em investigar coisas ou fatos e explicá-los segundo suas semelhanças e suas diferenças. Permite a análise de dados concretos e a dedução de semelhanças e divergências de elementos constantes, abstratos e gerais, propiciando investigações de caráter indireto.

Rey (2005) considera relevante nos diferentes momentos do processo da pesquisa a incorporação de outros aspectos que vão além dos processos matemáticos.

\subsection{Definição, Elaboração e Validação do Instrumento de Pesquisa}

No período de março a abril de 2020, foi aplicado um pré-teste da versão preliminar do questionário online, objetivando avaliar a eficácia da pesquisa e sanar possíveis dificuldades dos participantes. Estabeleceu-se um questionário composto por duas seções: a primeira de caráter geral sobre o perfil do hospital e aspectos específicos relacionados ao processo de coleta e transporte externo de resíduos biológicos e segunda sobre o perfil do respondente. A primeira seção foi composta por 17 questões, sendo 16 fechadas e uma aberta e a segunda por cinco questões fechadas. A consolidação dos dados em planilha Excel, somada às análises qualitativas realizadas, resultaram em um diagnóstico geral da atual situação dos serviços de coleta e transporte externo de RSS junto as regiões pesquisadas.

\subsection{Sobre a Abordagem feita aos Participantes e Aplicação da Pesquisa}

Foram realizados contatos telefônicos, junto as unidades hospitalares previamente mapeadas, para identificação dos responsáveis pelo gerenciamento de RSS. Identificado o profissional foi explicado o objetivo e a importância da participação de cada um para o mapeamento do cenário proposto pela pesquisa. Posteriormente, foram enviados e-mails contendo o link de acesso ao questionário online. De um total de 121 questionários respondidos, 35 aplicados na fase de pré-teste foram desconsiderados. As circunstâncias de realização da coleta de dados foram bastante desafiadoras, tendo em vista que o cenário de pandemia exigiu dos gestores atenção redobrada quanto as atividades de gerenciamento de RSS. 


\section{Resultados e Discussão}

\subsection{Perfil dos Hospitais}

Um total de 86 hospitais, sendo 72 privados e 14 públicos, foram considerados na consolidação dos resultados. Os hospitais públicos e privados de grande porte representaram 57\% da amostra, evidenciados na Tabela 1, com um total de 49 unidades. O perfil assistencial, considerando o porte da unidade e a natureza jurídica refletiu parte do cenário de saúde no Brasil. Os hospitais localizados na região Sudeste equivaleram a 80\%, com um total de 69 unidades, sendo 40 unidades em São Paulo, 22 no Rio de Janeiro e sete em Minas Gerais. A segunda maior representatividade foi a região Nordeste, com 11 hospitais. As demais regiões foram Centro-Oeste com 3\% do total, $2 \%$ na região Norte e 1\% no Sul. Evidenciou-se que mais da metade da amostra da pesquisa correspondeu a hospitais gerais (56\%), seguido dos especializados (23\%).

Tabela 1. Natureza jurídica e porte dos hospitais.

\begin{tabular}{l|c|c|c|c}
\hline Natureza jurídica & $\begin{array}{c}\text { Grande (151 } \\
\text { a 500 leitos) }\end{array}$ & $\begin{array}{c}\text { Médio (51 } \\
\text { a 150 leitos) }\end{array}$ & $\begin{array}{c}\text { Pequeno } \\
\text { (até 50 leitos) }\end{array}$ & Total \\
\hline Público (Estadual) & 6 & 2 & 1 & 9 \\
\hline Público (Federal) & 2 & 1 & & 3 \\
\hline Público (Municipal) & 1 & & 1 & 2 \\
\hline Privado (Fins lucrativos) & 30 & 22 & 2 & 54 \\
\hline Privado (Sem Fins lucrativos) & 10 & 6 & 2 & 18 \\
\hline Total & $\mathbf{4 9}$ & $\mathbf{3 1}$ & $\mathbf{6}$ & $\mathbf{8 6}$ \\
\hline
\end{tabular}

Fonte: Autores (2021).

\subsection{Perfil dos Respondentes}

Identificou-se que a maior parte dos profissionais responsáveis pelas atividades ligadas ao gerenciamento de RSS são do sexo feminino (79\%), conforme Figura 1. Quanto à idade dos profissionais, a pesquisa mostrou uma maioria de faixa etária entre 40 e 49 anos $(40 \%)$ e 31 e 39 anos de idade (35\%). Em relação à média do tempo de permanência, conforme apresentado na Figura 1, 30\% dos profissionais, estão há pelos menos três anos no hospital e $29 \%$ acima de dez anos. Outros $20 \%$ estão entre quatro e seis anos em seus postos de trabalho. A duração do vínculo empregatício com até um ano de trabalho correspondeu a 9\%, enquanto entre 7 e 9 anos, a $8 \%$ da amostra. Observou-se o setor de Hotelaria como a principal área responsável pelas atividades de gerenciamento de resíduos, equivalente a $73 \%$ dos respondentes. A equipe de Hotelaria em um ambiente hospitalar é o setor responsável por promover o conforto aos pacientes e acompanhantes, dando todo o suporte as áreas assistenciais no que se refere as condições da qualidade no atendimento, por meio das condições da acomodação, rouparia, higiene entre outras. Os resultados apontaram que tais atribuições ainda estão muito associadas ao sexo feminino, refletindo pouca diversidade de gênero. Boeger (2011) apresenta dados da pesquisa realizada pela Sociedade Brasileira de Hotelaria Hospitalar, no ano de 2009 com 200 hospitais, demonstrando que o perfil principal do gestor de hotelaria hospitalar é $85 \%$ formado por mulheres, seguido dos homens com $15 \%$.

Os profissionais com cargo de liderança corresponderam a $81 \%$ dos participantes, sendo o cargo de Supervisor/Coordenador com um total de 53 respondentes (62\%), seguido dos Gerentes com 17 profissionais (20\%). A ocupação do cargo de Analista e Técnico variou na faixa de 2\% a 3\%. Destaca-se que $8 \%$ dos pesquisados sinalizaram na descrição do cargo alguma referência a meio ambiente, tais como: Engenheira ambiental, Gerente de Sustentabilidade Ambiental, Supervisor e/ou coordenador ambiental, Técnico ambiental e/ou meio ambiente, analista ambiental e supervisor 
ambiental. Ao todo, um total de sete profissionais apresentaram essas descrições nos cargos, indicando possíveis mudanças no perfil dos profissionais que atuam com as atividades de gerenciamento de resíduos dentro dos estabelecimentos de saúde. Segundo Moura et al. (2011, p.2):

Apenas $10 \%$ dos hospitais brasileiros dispõem de recursos profissionais capacitados para atuar na área de Higiene Hospitalar, e $90 \%$ não tem enfermeiros especializados nem verbas para investir em materiais e equipamentos que possam minimizar os problemas gerados pelo lixo hospitalar.

Figura 1. Distribuição do perfil dos respondentes por gênero, faixa etária, tempo de atuação cargo.

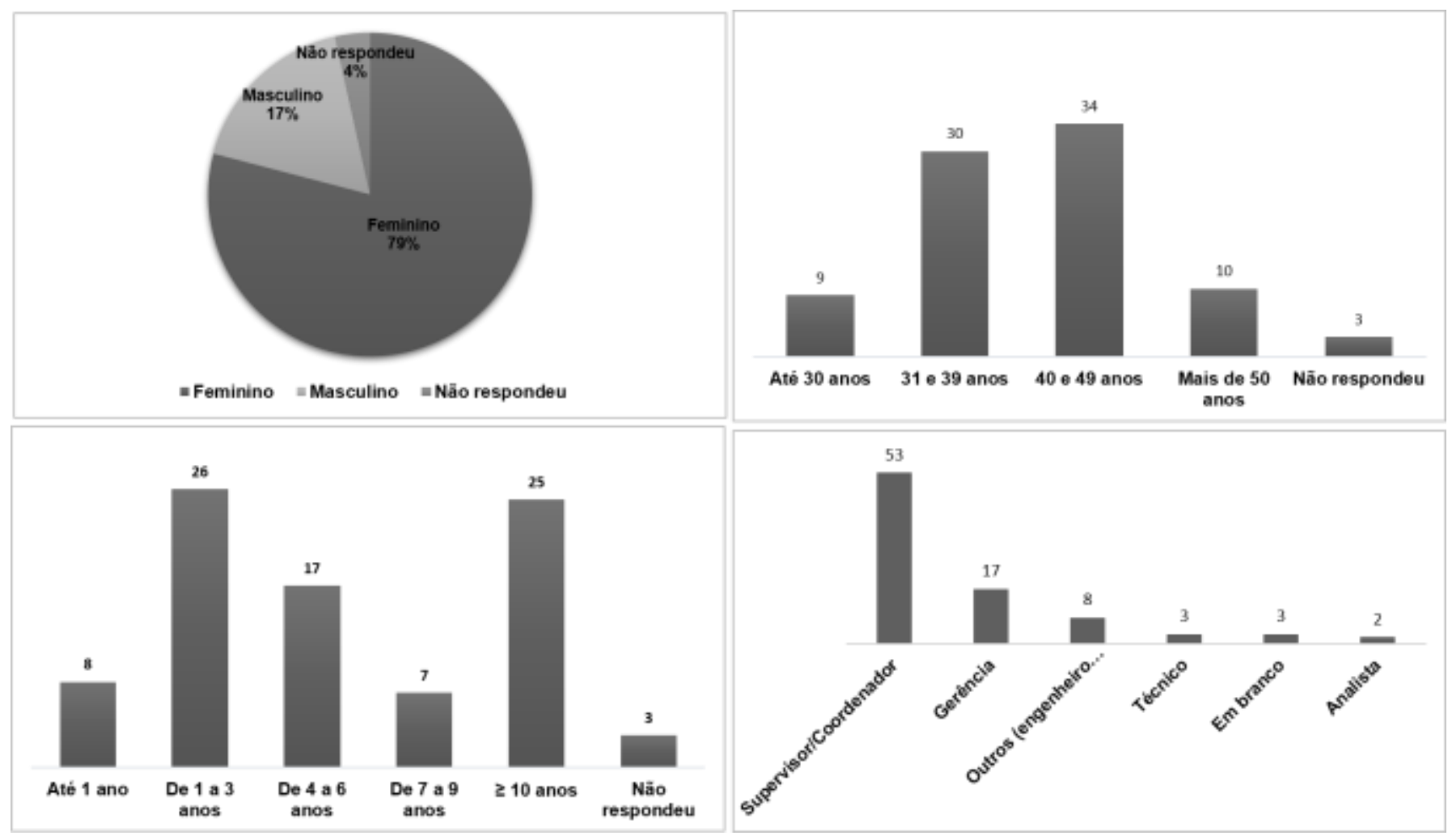

Fonte: Autores (2021).

\subsection{Análise dos serviços de coleta e transporte externos de resíduos biológicos}

Durante a realização da pesquisa, um fator de preocupação identificado entre os gestores foi o aumento significativo da geração de resíduos biológicos, como consequência do aumento do número de casos de contaminação por Covid-19, resultando em 2020 e 2021, na elevação da geração de resíduos biológicos, em comparação com 2019. Esse aumento de volume de RSS, em 2020, ainda não está refletido na publicação "Panorama dos Resíduos Sólidos do Brasil 2020" feita pela Associação Brasileira de Empresas de Limpeza Pública e Resíduos Especiais. No entanto, de acordo com a ABRELPE, a geração de lixo hospitalar no Brasil aumentou $20 \%$ no mês de junho de 2020 em comparação a igual período do ano passado. Segundo Bocchini (2020), "a geração média de lixo hospitalar por pessoa infectada e internada para tratamento de covid-19 tem sido de 7,5 quilos por dia”. Algumas variáveis devem ser levadas em consideração para avaliação do volume de resíduos gerados em uma instituição de saúde. Entre elas o porte do hospital, o perfil de atendimento, o tamanho etc. A Organização Mundial de Saúde aponta, em condições normais, em um hospital universitário são gerados de 4,1 a 8,7 kg leito, em um hospital geral, de 2,1 a 4,2 kg leito, e em ambulatórios de 0,5 a 1,8 kg (WHO, 1999). 
Dados publicados pela ABRELPE no Panorama dos Resíduos Sólidos do Brasil 2020 constatou, após pesquisa junto a 4.540 municípios brasileiros, que em 2019, foram coletadas 252.948 mil toneladas de Resíduos de Serviços de Saúde (RSS). Desse total, 91.477 toneladas, o equivalente a 36,2\% do volume total gerado, foram encaminhadas para lixões, valas sépticas e/ou aterros controlados (ABRELPE, 2018), conforme apresentado na Tabela 2. Por se tratar de local de disposição final de resíduos sem nenhuma preparação do solo ou adoção de mecanismos de prevenção da poluição, os lixões causam graves impactos ambientais como contaminação do solo e das águas superficiais e subterrâneas pelo chorume, proliferação de vetores de doenças e poluição do ar pela geração de gases poluentes, principalmente o metano (CH4), que é tóxico e altamente inflamável, e o dióxido de carbono $\left(\mathrm{CO}_{2}\right)$ que, juntamente com o metano e outros gases presentes na atmosfera, contribui para a intensificação do efeito estufa. Além de degradar a paisagem, produzir mau cheiro, colocar em risco o meio ambiente e a saúde pública, os lixões causam ainda impactos sociais negativos, tendo em vista que para muitas famílias a catação do lixo feita nestes locais em condições indignas e insalubres é o único meio de vida para sustento. Nesse sentido, os hospitais pesquisados demonstraram preocupação quanto a rastreabilidade dos resíduos gerados. O equivalente a $85 \%$ afirmou que a emissão do Manifesto de Transporte de Resíduos (MTR), documento que contém todas as informações necessárias dos resíduos que serão transportados até a sua disposição final ambientalmente correta é parte integrante do gerenciamento dos RSS. Um percentual de 12\% respondeu não realizar a emissão diária do MRT e outros 3\% não responderam. A Portaria MMA n 280 (DOU de 30/06/2020 Seção I Pág. 95), instituiu em todo o território nacional o Manifesto de Transporte de Resíduos nacional feito de forma on-line (BRASIL, 2020). Desde $1^{\circ}$ de janeiro de 2021 , esse requisito se tornou obrigatório para todos os geradores de resíduos sujeitos à elaboração de Plano de Gerenciamento de Resíduos Sólidos, conforme disposto no Art. 20 da Lei $\mathrm{n}^{\circ} 12.305$, de 2 de agosto de 2010.

Tabela 2. Quantidade anual de RSS coletados por região e tipo de tratamento empregado.

\begin{tabular}{|c|c|c|c|c|c|c|c|c|c|}
\hline \multirow[b]{2}{*}{ Região } & \multirow[b]{2}{*}{$\begin{array}{c}\text { Total } \\
\text { RSS } \\
\text { Gerado }\end{array}$} & \multicolumn{2}{|c|}{ Autoclave } & \multicolumn{2}{|c|}{ Incineração } & \multicolumn{2}{|c|}{ Microondas } & \multicolumn{2}{|c|}{ Outros } \\
\hline & & $\begin{array}{r}\text { Total } \\
\text { RSS } \\
\text { Tratado } \\
\end{array}$ & $\%$ & $\begin{array}{r}\text { Total } \\
\text { RSS } \\
\text { Tratado }\end{array}$ & $\%$ & $\begin{array}{r}\text { Total } \\
\text { RSS } \\
\text { Tratado } \\
\end{array}$ & $\%$ & $\begin{array}{r}\text { Total RSS } \\
\text { Tratado }\end{array}$ & $\%$ \\
\hline Norte & 9.582 & 134 & $1,40 \%$ & 4.561 & $47,60 \%$ & 0 & $0,00 \%$ & 4.887 & $51,00 \%$ \\
\hline Nordeste & 36.554 & 3.217 & $8,80 \%$ & 20.799 & $56,90 \%$ & 0 & $0,00 \%$ & 12.538 & $34,30 \%$ \\
\hline $\begin{array}{l}\text { Centro- } \\
\text { Oeste }\end{array}$ & 18.451 & 4.299 & $23,30 \%$ & 12.030 & $65,20 \%$ & 0 & $0,00 \%$ & 2.122 & $11,50 \%$ \\
\hline Sudeste & 175.775 & 32.518 & $18,50 \%$ & 58.885 & $33,50 \%$ & 12.656 & $7,20 \%$ & 71.716 & $40,80 \%$ \\
\hline Sul & 12.586 & 6.645 & $52,80 \%$ & 5.437 & $43,20 \%$ & 289 & $2,30 \%$ & 214 & $1,70 \%$ \\
\hline Total & 252.948 & 46.814 & $18,5 \%$ & 101.712 & $40,2 \%$ & 12.945 & $5,1 \%$ & 91.477 & $36,2 \%$ \\
\hline
\end{tabular}

Fonte: Autores (2021).

Nas etapas intraestabelecimento do gerenciamento de RSS, observou-se na pesquisa conformidade com a legislação. Mais de $90 \%$ dos participantes responderam que mantêm os sacos de lixo biológicos no Abrigo Externo devidamente acondicionados dentro dos contêineres para o momento da coleta externa. A prática está em consonância com o art. 27 da RDC no. 222/2018/ANVISA onde estabelece que: “... no armazenamento temporário e externo de RSS é obrigatório manter os sacos acondicionados dentro de coletores com a tampa fechada" (ANVISA, 2018, p.11). Três hospitais privados, o equivalente a 3\%, responderam que o acondicionamento dos sacos de lixo biológicos no Abrigo Externo é feito em cima de pallets ou diretamente sobre os pisos. Segundo a ANVISA (2018), a contenção adequada deve ser o principal instrumento de proteção à saúde do trabalhador. Manter os sacos de lixo sob paletes torna o processo mais vulnerável. Isso acontece porque demandará do coletor, tanto interno quanto externo, o uso contínuo e intenso das mãos, braços e pernas para levantamento dos sacos, com distanciamento adequado do corpo. A NR 9 no item 9.3.5.1 especifica que: (SERPT, 1978, p.3) "Deverão ser adotadas as 
medidas necessárias suficientes para a eliminação, a minimização ou o controle dos riscos ambientais sempre que forem verificadas uma ou mais das seguintes situações".

O equivalente a $52 \%$ da amostra, 45 hospitais, responderam que a retirada dos resíduos biológicos do Abrigo Externo para fins de transporte externo ocorre de forma conteinerizada, ou seja, sem nenhum contato manual com os sacos de lixo, em conformidade com a legislação. No entanto, um percentual de 47,7\%, correspondente a 41 hospitais apontaram inconformidades no processo de retirada dos resíduos biológicos, principalmente relacionadas a coleta manual, ou seja, tendo contato direto com os sacos de lixo. A coleta manual, sem os mecanismos adicionais de proteção, deixa a equipe de coleta externa mais suscetíveis a riscos de acidentes. No caso da coleta manual, tal vulnerabilidade se estende nas unidades de tratamento dos resíduos biológicos, tendo em vista que os sacos serão retirados manualmente por parte do profissional antes de serem encaminhados para o tratamento. Segundo Silva et al. (2014, p.7) "esse tipo de procedimento de coleta acarreta o aumento do risco de ocorrer um acidente com o funcionário, caso tenha RSS dispostos inadequadamente (principalmente perfurocortantes)". Apesar dos resíduos perfurocortantes representar uma parcela menor dos RSS, segundo Sinoti et al (2009) o equivalente a $1 \%$ do volume de RSS gerados em um ambiente hospitalar, se descartados de forma inadequada, constitui-se em maior risco à saúde do trabalhador.

Os serviços de saúde apresentam uma significativa relação na transmissão do vírus da hepatite B e uma delas pode ser decorrente de acidentes com resíduos perfurocortantes. Segundo Mol (2016, p.30), “o risco de infecção pelos vírus das hepatites B ou C pode estar associado aos casos de acidentes com resíduos perfurocortantes, através de cortes ou perfurações com material contaminado". A utilização de luvas pelo trabalhador atenua, mas não impede a maior parte dos acidentes, que não atingem apenas as mãos, mas também braços e pernas (Ferreira \& Anjos, 2001).

Diversos autores (Oliveira et al., 2021, Rocha et al., 2021, Delevati et al., 2019; Gomes et al., 2019, Uehara et al., 2019; Accurio et al., 1998; Anjos et al., 1995; Cantanhede, 1997; Diaz et al., 1997; Ferreira, 1997; Leite; Lopes, 2000; Maglio, 2000; Robazzi et al., 1992; Velloso, 1995; Zepeda, 1995) reforçam os aspectos de riscos inerentes ao gerenciamento de resíduos. Ferrão et. al. (2021); Bleck e Wettberg (2012) apontam que as condições de trabalho e o meio ambiente influenciam no aparecimento dos riscos.

Segundo a ANVISA (2018), o descarte dos resíduos perfurocortantes deve ser feito em recipientes identificados, rígidos, providos com tampa, resistentes à punctura, ruptura e vazamento. A norma ABNT NBR 12810:2020, que trata do gerenciamento extraestabelecimento de resíduos de serviços de saúde e a ABNT NBR 13853-1:2018 Versão Corrigida:2020 define que a confecção das caixas coletoras de perfurocortantes deve resistir ao corte e a perfuração pelos objetos contidos em seu interior e devem ser à prova de vazamentos. No Brasil, entre os modelos de caixas coletoras para os resíduos perfurocortantes estão as caixas especiais de papelão, revestidas internamente com saco plástico visando proteger contra vazamentos de fluidos contaminados. Evidenciou-se na presente pesquisa que o uso de modelos constituídos de papelão, com a necessidade de montagem, não é prática comum em outros países para o acondicionamento de materiais perfurocortantes. De acordo com estudos feitos pela equipe do Projeto Hospitais Saudáveis, ponto focal no Brasil da organização internacional Health Care Without Harm (HCWH) - Saúde sem Dano (SSD), identificou-se que nos EUA, Japão e Alemanha, os modelos das caixas coletoras de resíduos perfurocortantes são fabricados em plástico rígido de alta resistência, com a opção de desconector para o descarte seguro de agulhas. Alguns desses modelos de coletores para materiais perfurocortantes fabricados em plástico rígido estão disponíveis no mercado brasileiro, no entanto, mais onerosos. Outro diferencial observado no descarte de resíduos perfurocortantes no Brasil em relação aos países citados acima está na prática em relação aos procedimentos operacionais para coleta e transporte externo de RSS. Nos hospitais brasileiros pesquisados identificou-se, como prática predominante, que os coletores de materiais perfurocortantes após preenchidos em sua capacidade são fechados, lacrados e acondicionados em sacos brancos leitosos com a simbologia de risco biológico. Posteriormente, esses sacos de resíduos 
biológicos são colocados dentro dos contêineres ao Abrigo Externo. Na Tabela 3, há indicação de que uma parcela significativa, o equivalente a 40\%, os sacos de resíduos biológicos, inclusive com a presença de caixas de perfurocortantes, vão soltos dentro do caminhão, sem nenhum mecanismo adicional de proteção, portanto sem garantia quanto a preservação das condições de acondicionamento até a destinação final.

Tabela 3. Forma de transporte dos resíduos no caminhão.

\begin{tabular}{l|c|c}
\hline $\begin{array}{l}\text { Como os sacos de lixo de resíduos infectantes são transportados até as unidades } \\
\text { de tratamento? }\end{array}$ & Total & $\%$ \\
\hline $\begin{array}{l}\text { Em contêineres, bombonas e/ou tambores em boas condições de uso e devidamente } \\
\text { identificados no interior do caminhão (modelo carroceria fixa). Os sacos são } \\
\text { mantidos dentro de recipientes no interior do caminhão. }\end{array}$ & 37 & $43 \%$ \\
\hline $\begin{array}{l}\text { Diretamente no interior do caminhão, ou seja, sacos dispostos fora de recipientes. } \\
\text { Os sacos de lixo vão soltos dentro do caminhão modelo carroceria fixa }\end{array}$ & 34 & $40 \%$ \\
\hline Diretamente no caminhão após basculamento (modelo caminhão compactador) & 13 & $15 \%$ \\
\hline Outros (não soube informar) & 2 & $2 \%$ \\
\hline
\end{tabular}

Fonte: Autores (2021).

Delevati et al. (apud Silva et al., 2019, p.6), cita os resultados de uma pesquisa feita junto a 34 municípios da região metropolitana de Belo Horizonte, onde avaliou-se um total de 54 estabelecimentos de saúde e identificou-se inconformidades quanto ao atendimento aos requisitos legais, entre eles ligados ao local de armazenamento dos resíduos. Segundo Gomes et al. (2019), a ocorrência de acidentes de trabalho com material biológico tem envolvido não apenas os profissionais de saúde, mas também os trabalhadores que atuam nos serviços de higiene e limpeza dos hospitais, coletores externos e de limpeza pública e os catadores de materiais recicláveis. O fato gerador está no descarte incorreto de resíduos. Foi identificado que o uso de contêineres rígidos e resistentes na etapa de transporte externo de resíduos de serviços de saúde têm sido a prática em diversos países e mesmo em diferentes regiões do Brasil, no entanto faz-se necessário padronizar esses dispositivos no sentido de garantir seu desempenho (Figura 4). "Na maior parte do Brasil, onde não se usa a conteinerização, a contenção depende exclusivamente da integridade dos sacos plásticos, que são submetidos a diversos esforços e impactos ao longo do manejo dos resíduos (Ribeiro Filho, 2012)”.

Estudos de Rafaela et al. (2013) demostram que os trabalhadores percebem o risco ao manipularem os RSS, visto que direcionam maior atenção ao grupo E, perfurocortantes, no entanto, não estão cientes quanto aos demais riscos decorrentes do gerenciamento ineficaz dos RSS.

Xavier et al. (2010) em pesquisa sobre a exposição dos trabalhadores a material biológico no manejo externo dos resíduos identificou que a maioria dos acidentes se originou do contato direto com o lixo, principalmente durante a transposição deste do abrigo dos estabelecimentos de saúde para o caminhão de coleta e do caminhão ao aterro sanitário.

Lopes et al. (2012) aponta que a rotatividade entre os trabalhadores que atuam nos serviços de limpeza urbana é extremamente elevada, dificultando a realização de programas treinamento e de prevenção, contribuindo desta maneira para o aumento no número de acidentes. Uma pesquisa desenvolvida por Silva et al. (2013), cujos aspectos técnicos ligados ao gerenciamento de resíduos de serviços de saúde também foram estudados por Silva, Sperling e Barros (2014), identificou falhas importantes no gerenciamento de resíduos, sendo alguns deles relacionados aos comportamentos por parte dos funcionários de coleta. Foram citados como exemplos de comportamento de riscos o carregamento simultâneo de vários sacos, dificultando mantê-los distantes do corpo no momento da coleta e a utilização de cabos de vassoura ou materiais que possam furar os sacos para retirada do ar, permitindo assim maior compactação dos sacos dentro veículos. Tal prática contraria a NR 
32, onde especifica que o transporte manual do recipiente de segregação deve ser realizado de forma que não exista o contato do mesmo com outras partes do corpo, sendo vedado o arrasto.

Segundo Ribeiro Filho (2012) os sacos plásticos usados no acondicionamento dos RSS biológicos cumprem adequadamente a função de contenção dos agentes perigosos presentes nesses resíduos, no entanto, suas propriedades não oferecem a resistência mecânica necessária para assegurar a integridade desses sacos ao longo das etapas de transporte e armazenagem. No entendimento de Ribeiro Filho (2012), a proposição de uma regulamentação do uso de contêineres no acondicionamento dos RSS a fim de garantir a proteção mecânica da embalagem inicial, proporciona mais proteção aos trabalhadores que realizam o manejo interno e externo dos resíduos, da população e do meio ambiente, pois garante a preservação das condições de acondicionamento até a destinação final. $\mathrm{O}$ art. $9^{\circ}$, parágrafo único, da Resolução $\mathrm{n}^{\circ}$ 358/2005/CONAMA cita que "as características originais de acondicionamento devem ser mantidas, não se permitindo abertura, rompimento ou transferência do conteúdo de uma embalagem para outra" (CONAMA, 2005, p.2). Essa afirmativa também é evidenciada na NR 32, especificando que os sacos plásticos utilizados no acondicionamento dos resíduos de saúde devem atender ao disposto na ABNT NBR 9191 e ainda ser mantidos íntegros até o tratamento ou a disposição final do resíduo. Na análise dos resultados, observou-se que o entendimento de coleta conteinerizada compreendida pelos participantes da pesquisa consiste no modelo de caminhão que possui braços mecânicos que elevam os contêineres e despejam os sacos de lixo em seu interior. O que não está incorreto, caso não fosse utilizado para a coleta e transporte dos resíduos biológicos.

A Tabela 4 apresenta os tipos de veículos utilizados na coleta e transporte externo de resíduos biológicos praticados junto aos hospitais brasileiros, sendo o uso de caminhão de carroceria fixa o mais comum para os resíduos biológicos, a exceção fica por conta dos hospitais localizados em São Paulo, capital, onde identificou-se como prática o uso de caminhão compactador com a técnica de basculamento. A prática do basculamento dos resíduos para um caminhão compactador é autorizada pela ANVISA e demais órgãos competentes apenas para os resíduos classificados como comuns, que são similares aos domésticos (BRASIL, 2018). Tal medida se aplica em razão do risco dos sacos de lixo rasgarem e ocorrer o vazamento dos resíduos, com um potencial de contaminação do meio ambiente e dos trabalhadores envolvidos no processo de gerenciamento dos RSS. Nove hospitais de grande porte sinalizaram que as empresas contratadas procedem à retirada dos resíduos biológicos por meios mecânicos, procedendo ao basculamento dos sacos de lixo dos contêineres para o caminhão compactador. O uso de caminhão compactador para a coleta de resíduos biológicos (Grupo A e Grupo E) contraria a condição estabelecida no art. 38 da RDC 222/2018/ANVISA, tendo em vista a necessidade de integridade dos sacos de lixo durante todas as etapas do gerenciamento. A determinação do órgão regulador é a de que os veículos de transporte de resíduos biológicos não contenham sistemas de compactação. "Os veículos de transporte externo dos RSS não podem ser dotados de sistema de compactação ou outro sistema que danifique os sacos contendo os RSS, exceto para os RSS do Grupo D” (BRASIL, 2018).

Tabela 4. Avaliação da terceirizada - Tipos de veículos usados na coleta e transporte externo de RSS.

\begin{tabular}{|c|c|c|c|c|c|c|c|c|c|c|}
\hline \multirow{2}{*}{ Veículo } & \multicolumn{9}{|c|}{ Estado } & \multirow{2}{*}{$\begin{array}{l}\text { Total } \\
\text { Geral }\end{array}$} \\
\hline & $\mathbf{A M}$ & BA & DF & MA & MG & PR & PE & $\mathbf{R J}$ & SP & \\
\hline $\begin{array}{l}\text { Caminhão baú com } \\
\text { carroceria fixa fechada }\end{array}$ & 2 & 7 & 3 & 1 & 7 & 1 & 3 & 21 & 16 & 61 \\
\hline Caminhão compactador & & & & & & & & 1 & 22 & 23 \\
\hline Caminhão tanque & & & & & & & & & 1 & 1 \\
\hline $\begin{array}{l}\text { Em carro tipo } \\
\text { furgão/fiorino fechada. }\end{array}$ & & & & & & & & & 1 & 1 \\
\hline
\end{tabular}

Fonte: Autores (2021). 
A variável ambiental cada vez mais está incorporada na gestão das empresas. Não se trata apenas de um diferencial competitivo, mas sim uma questão de sobrevivência em longo prazo. A população em decorrência de sua consciência ambiental, que gradativamente vem ganhando mais espaço, passou a demandar por produtos e processos produtivos com menos impacto no meio ambiente. Assim, as questões ambientais passaram a ganhar atenção crescente das organizações internacionais, dos governos, das empresas e da sociedade. Essa nova postura corporativa tem proporcionado a materialização e a aplicação de diversos mecanismos, objetivando não apenas detectar e mensurar possíveis problemas, como também o oferecimento de alternativas de soluções. Entre esses mecanismos, destacam-se as políticas de gestão de riscos relacionadas à auditoria ambiental, um instrumento que permite avaliar o sistema de gestão ambiental, o grau de implementação e a eficiência dos planos e programas no controle da poluição ambiental, a conformidade legal e o controle ambiental de empreendimentos que exercem atividades potencialmente poluidoras.

Evidenciam-se no mercado, grandes empresas privadas, preocupadas com a responsabilidade ambiental e que compartilham com seus fornecedores essas questões, condicionando suas compras e contratações a critérios socioambientais bem definidos. Algumas empresas passaram a aplicar formulários sobre questões ambientais a seus fornecedores ou a realizar auditorias visando avaliar sua gestão ambiental, com risco de suspensão de um contrato caso o fornecedor não atenda a requisitos mínimos desejados. Esse é inclusive um dos critérios para que uma empresa mantenha sua certificação na norma ABNT NBR ISO 14001, que é influenciar positivamente um fornecedor para que melhore sua atuação ambiental.

Um total de 36 hospitais (42\%) informou realizar auditorias ambientais uma vez por ano, contra 17 (20\%) que não estão realizando e 13 que realizam esporadicamente. Apenas 14 hospitais (16\%) informaram realizar duas vezes por ano auditorias ambientais junto aos prestadores de serviços de coleta e transporte externo de RSS. As auditorias ambientais permitem avaliar objetivamente cada aspecto ambiental relevante a ser considerado na contratação de uma empresa especializada em transporte de cargas perigosas. São exemplos: a regularidade da empresa com as determinações da Política Nacional de Resíduos Sólidos - Lei n 12.305/2010; a destinação do óleo lubrificante usado conforme a Resolução CONAMA 362/2005; limpeza e manutenção regulares da drenagem dos diques de lavagem de veículos, bem como das caixas separadoras de água/óleo e tanques de acúmulo interligados ao sistema; manutenção dos veículos realizada apenas sobre o piso impermeável da oficina; método de higienização dos veículos; e instalações do pátio de estacionamento de frota de caminhões em conformidade com as diretrizes da lei de zoneamento do município e as recomendações e condicionantes estabelecidas em leis específicas para o uso do solo, código de edificação, posturas, vigilância sanitária, corpo de bombeiros e defesa civil.

As empresas de Transporte de Rodoviário de Cargas Perigosas devem manter um Plano de Atendimento a Emergências (PAE) adequado aos aspectos ambientais e os impactos relacionados aos Perigos e Riscos decorrentes de suas atividades, bem como realizar treinamentos periódicos com o objetivo de que todos os envolvidos tenham conhecimento do referido plano de emergência em sua totalidade. O documento deve ser atualizado anualmente ou quando houver mudanças significativas e deverá ser apresentado ao órgão competente para análise. Uma porcentagem significativa de respondentes (53\%) sinalizou desconhecimento acerca do Plano de Emergência da empresa contratatada para os serviços de coleta e transporte externos de resíduos de serviços de saúde. O equivalente a $47 \%$ afirmou ter conhecimento acerca do documento.

A CETESB define o PAE como:

...um conjunto de diretrizes, dados e informações que propiciem as condições necessárias para a adoção de procedimentos lógicos, técnicos e administrativos, estruturados para serem desencadeados rapidamente em situações de emergência, para a minimização de impactos à população e ao meio ambiente [CETESB,1981].

O Plano de Emergência para ser eficaz não deve ser visto como um documento burocrático, organizado e bem escrito. Ele é um guia prático e deve ser conhecimento de todos os envolvidos. Sua elaboração implica em conhecer previamente as 
situações de emergência que podem ocorrer e os possíveis danos associados. Deve evidenciar os fluxos de comunicação em condições de emergência ou crise na área de Meio Ambiente. Na Tabela 5, os resultados apontaram que mais de 70\% dos hospitais participantes da pesquisa possuem implementados em suas rotinas de trabalho procedimentos preliminares de avaliação de riscos como o acompanhamento diário das coletas de resíduos e avaliações periódicas das licenças ambientais. Embora grande parte dos pesquisados confirmou realizar o acompanhamento das coletas externas diariamente, as visitas técnicas regulares junto aos prestadores de serviços ligados de coleta e transporte externos de resíduos biológicos está presente em apenas $26 \%$ dos pesquisados. O número de respostas relacionadas a baixa adesão quanto à frequência de visitas técnicas para avaliação da conformidade dos processos relativos as etapas externas do gerenciamento de RSS foram de 57\%. De um total de 86 hospitais pesquisados, 49 realizam visitas técnicas apenas ocasionalmente e outras 13 unidades nunca procederam. Apenas 22 hospitais confirmaram realizar visitas técnicas com regularidade.

Segundo os fundamentos da Resolução CONAMA nº 306/2002, as auditorias ambientais permitem avaliar o sistema de gestão ambiental, o grau de implementação e a eficiência dos programas no controle da poluição ambiental, a conformidade legal e o controle ambiental dos empreendimentos que exercem atividades potencialmente poluidoras, como o caso das empresas ligadas as atividades de transporte de resíduos perigosos. Assim, as visitas técnicas ou as auditorias ambientais são extremamente importantes, pois as evidências quanto à conformidade legal estão fundamentadas não apenas em análise documental, mas também em inspeções nas instalações, devendo ser observado principalmente os aspectos ambientais significativos, como por exemplo, os sistemas de controle de poluição do ar e da água.

Quando questionado sobre a realização de avaliação do nível de serviço, objetivando identificar distorções relevantes de conformidade em relação as cláusulas contratuais, os resultados entre as instituições de saúde que sempre realizam contra os que procedem apenas ocasionalmente se equipararam. Conforme evidenciado na Tabela 5, o equivalente a $42 \%$ informou realizar de forma sistemática e disciplinada e outros $42 \%$ de apenas forma ocasional. Em relação a avaliação global dos gestores quanto a qualidade dos serviços prestados pelas contratadas de coleta e transporte de resíduos de serviços de saúde um percentual de $93 \%$ se mostrou favorável, sendo 64 responderam como bom e 16 como excelente. Apenas $2 \%$ dos pesquisados classificou os serviços como ruim e outros $5 \%$ como regular.

Tabela 5. Avaliação da terceirizada.

\begin{tabular}{lcccc}
\hline Atividades & Sempre & Ocasionalmente & Nunca & Em branco \\
\hline Acompanhamento diário das coletas & 63 & 23 & & \\
Avaliações periódicas das licenças ambientais & 66 & 18 & 1 & 1 \\
Visitas técnicas & 22 & 49 & 13 & 2 \\
Avaliação do nível de serviço & 36 & 36 & 11 & 3 \\
Conservação dos veículos & 53 & 26 & 4 & 3 \\
\hline
\end{tabular}

Fonte: Autores (2021).

Os Princípios de Direito Ambiental, como o do Poluidor Pagador e de previsões legais expressas como a do $\S 1^{\circ}$ do artigo 14 da Política Nacional do Meio Ambiente, Lei 6.938/81 especifica que "é o poluidor obrigado, independentemente da existência de culpa, a indenizar ou reparar os danos causados ao meio ambiente e a terceiros, afetados por sua atividade". O artigo 225 da Constituição Federal de 1988, no $§ 3^{\circ}$ estabelece que:

As condutas e atividades consideradas lesivas ao meio ambiente sujeitarão os infratores, pessoas físicas ou jurídicas, a sanções penais e administrativas, independentemente da obrigação de reparar os danos causados (BRASIL, 1988). 
Além dos riscos ao meio ambiente, as instituições de saúde nas auditorias devem atentar-se quanto aos aspectos de segurança ocupacional dos trabalhadores da empresa contratada. Os resultados da pesquisa apontaram que um total de 72 respondentes $(84 \%)$ citaram o uso adequado dos EPIs por parte dos funcionários da empresa contratada. O equivalente a 12 respondentes (14\%) citou que tal procedimento ocorre as vezes e dois não souberam informar. As medidas de prevenção compreendem um conjunto de ações, tratados no decorrer da presente pesquisa e que vão além do uso adequado dos EPIs por parte dos trabalhadores. Os mecanismos adequados e seguros de contenção dos resíduos de serviços de saúde em todas as etapas do processo de gerenciamento dos resíduos são fundamentais para a garantia da saúde do trabalhador e possíveis impactos ao meio ambiente. Ferreira e Anjos (2001) apontaram que o uso de luvas por parte do trabalhador atenua, mas não impede acidentes, pois outras partes do corpo como braços e pernas também podem ser atingidos. Nas visitas técnicas, as entrevistas são um importante instrumento de medição da regularidade da empresa perante os aspectos legais relacionados a saúde e segurança ocupacional. Questões ligadas aos programas de treinamento, capacitação técnica dos responsáveis pela operação e manutenção dos sistemas, rotinas, instalações e equipamentos de proteção ao meio ambiente ou que possuem o potencial de causar danos ambientais e medidas preventivas simples como campanhas gestão do trabalho focada no risco e outras formas de segurança devem ser observados. As ações de acompanhamento, monitoramento e avaliação das atividades de coleta interna e externa devem ser ainda mais intensificadas.

\section{Conclusão}

Os sistemas de contenção adotados pelos hospitais nas etapas intraestabelecimento do processo de gerenciamento de RSS biológicos não são mantidos até as unidades de tratamento. O total de 41 hospitais, equivalente a 47,7\%, apontaram inconformidades no processo de coleta externa. São exemplos a retirada manual dos sacos de lixo dos contêineres de resíduos do Abrigo Externo e transporte sem nenhum mecanismo adicional de proteção, sendo estes levados soltos diretamente no interior do caminhão modelo carroceria fixa e o basculamento dos sacos de lixo para caminhões compactadores. Tais resultados respondem a um dos objetivos específicos da pesquisa que foi o de mapear como os RSS biológicos são retirados dos hospitais e transportados até as áreas de transbordo ou unidades de tratamento antes da disposição final ambientalmente adequada.

Acerca de como os gestores hospitais monitoram a conformidade legal das empresas contratadas para a coleta e transporte externos de RSS biológicos, verificado que mais de 70\% dos gestores procedem ao controle por meio das avaliações periódicas das licenças ambientais e acompanhamento diário das coletas. No entanto, os resultados apontaram baixa frequência da realização de visitas técnicas e/ou auditorias ambientais externas, impossibilitando avaliações periódicas quanto o grau de implementação e a eficiência dos planos e programas no controle da poluição ambiental. Identificado desconhecimento por parte dos gestores quanto a obrigatoriedade do Plano de Emergência para os serviços de coleta e transporte externos de RSS como parte integrante de um Programa de Gerenciamento de Riscos. Os resultados apontaram satisfação dos gestores quanto a qualidade dos serviços prestados pelas empresas de coleta e transporte externos de RSS biológicos contratados.

Nas etapas extraestabelecimento, observou-se junto aos hospitais pesquisados que os atuais mecanismos adotados pelas terceirizadas fragiliza a garantia de que os sacos de lixo permaneçam íntegros durante todas as etapas do processo de gerenciamento, expondo a saúde do trabalhador. Identificou-se oportunidades de melhorias quanto ao acompanhamento por parte dos gestores no que tange as etapas extraestabelecimento. Tal constatação se deu em virtude do desconhecimento dos gestores quanto a existência de Planos de Emergência por parte das terceirizadas e pouca frequência de realização de auditorias ambientais como principal ferramenta de acompanhamento. Os resultados da pesquisa apontaram fragilidades em relação ao conhecimento do Plano de Emergência da empresa contratada, sendo a obrigatoriedade desconhecida por 53\% dos 
respondentes. Admitindo-se que sejam as pessoas mais qualificadas dentro da cadeia de custódias, esse conhecimento deveria ser priorizado pelas empresas contratadas e pelos estabelecimentos de saúde.

Os hospitais recebem e tratam as consequências dos desequilíbrios ambientais e sociais, mas também geram impactos ao prestar seu serviço, pois consomem recursos e geram resíduos durante 24 horas por dia. Considerando o conceito de saúde única, termo trata da integração entre a saúde humana, a saúde animal e o ambiente, a gestão ambientalmente adequada dos resíduos de serviços de saúde tem um papel fundamental na mitigação das ameaças à saúde pública, como a proliferação de vetores, microorganismos patogênicos, agentes químicos etc. causados por inconformidades na gestão. Dessa forma, para estudos futuros, recomenda-se aprofundar pesquisas relacionadas a quais estratégias e diretrizes os hospitais têm estabelecido para contribuir com a minimização da geração de resíduos, visando aumentar a quantidade e a qualidade dos materiais separados para reciclagem, diminuindo, assim, a sobrecarga de resíduos enviados para os aterros sanitários, e em outros casos os lixões, e promover a educação ambiental.

\section{Referências}

Acurio, G., Rossin, A., Teixeira, P. F., \& Zepeda, F. (1997). Diagnóstico de la situación del manejo de resíduos sólidos municipales em América Latina y el Caribe. Banco Interamericano de Desenvolvimento, Organização Panamericana da Saúde.

Alves, S. B., Souza, A. C. S., Tipple, A. F. V., Rezende, K. C. D., Rezende, F. R. \& Rodrigues, E. G. (2012). Manejo de resíduos gerados na assistência domiciliar pela estratégia de saúde da família. Revista Brasileira de Enfermagem, 65(1):128-134.

Anjos, L. A.; Barros, A. A.; Ferreira, J. A.; Oliveira, T. C. E.; Severino, K. C.; Silva, M. O. \& Waissmann, W., (1995). Gasto Energético e Carga Fisiológica de Trabalho em Coletores de Lixo Domiciliar no Rio de Janeiro: Estudo Piloto Rio de Janeiro: Escola Nacional de Saúde Pública, Centro de Estudos de Saúde do Trabalhador e Ecologia Humana. (mimeo.)

Associação Brasileira de Normas Técnicas (2008). NBR 9191. Sacos plásticos para acondicionamento de lixo: Requisitos e métodos de ensaio. Rio de Janeiro.

Associação Brasileira de Empresas de Limpeza Pública e Resíduos Especiais. (2020). Panorama dos Resíduos Sólidos no Brasil. https://abrelpe.org.br/panorama/

Associação Brasileira de Normas Técnicas (2020). NBR 12810. Resíduos de serviços de saúde, Gerenciamento extraestabelecimento, Requisitos. Rio de Janeiro.

Associação Brasileira de Normas Técnicas (2021). NBR 13221. Transporte terrestre de produtos perigosos. Resíduos. Rio de Janeiro.

Brasil. (2018). Agência Nacional de Vigilância Sanitária. Resolução da Diretoria Colegiada $n^{o} 222$, de 28 de março de 2018. Dispõe sobre o Regulamento Técnico para o gerenciamento de Resíduos de Serviços de Saúde. https://www.gov.br/anvisa/ptbr/centraisdeconteudo/publicacoes/servicosdesaude/publicacoes/rdc-222-de-marco-de-2018-comentada.pdf/view.

Brasil. (2005). Conselho Nacional do Meio Ambiente. Resolução no 358, de 29 de abril de 2005. Dispõe sobre o tratamento e a disposição final dos resíduos $\operatorname{dos}$ serviços de saúde e da outra hrovidencias. /legiabre.cfm?codlegi=462.

Brasil. Lei. 12.305 de 2 de agosto de 2010. Institui a Política Nacional de Resíduos Sólidos; altera a Lei n 9.605 , de 12 de fevereiro de 1998 ; e dá outras providências. http://www.planalto.gov.br/ccivil_03/_ato2007-2010/2010/lei/112305.htm

Bleck, D. \& Wettberg, W. (2012). Waste collection in developing countries-Tackling occupational safety and health hazards at their source. Waste management,32(11):2009-2017.

Boeger, M. (2020). Hotelaria Hospitalar: gestão em hospitalidade e humanização. Editora Senac São Paulo.

Cantanhede, A. (1997). Experiences from the Pan-American Centre of Sanitary Engineering \& Environmental Sciences-Difficulties and possibilities, in: Latin American-Swedish Seminar on Solid Waste Management, Proceedings. Rio de Janeiro: Associação Brasileira de Engenharia Sanitária e Ambiental/Lund University.

Companhia de Tecnologia de Saneamento Ambiental (CETESB). (2021). Plano de ação de emergência - PAE. https://cetesb.sp.gov.br/emergenciasquimicas/tipos-de-acidentes/rodovias/plano-de-acao-de-emergencia-pae/

Confederação Nacional de Saúde, Hospitais, Estabelecimentos e Serviços (CNSaúde). (2019). Cenário dos hospitais no Brasil. http://cnsaude.org.br/wpcontent/uploads/2019/05/CenarioDosHospitaisNoBrasil2019CNSaudeFBH.pdf

Delevati, D. S., Castro, M. M. R. S., Ries, E. F., Bayer, V. M. L. \& Rocha, V. M. P. (2020). Desafios na gestão de resíduos de estabelecimentos de saúde públicos perante a RDC 222/18. Saúde em Debate, 43(3):190-199, 2019.

Dias, M. A. A. (2004). Resíduos dos Serviços de Saúde e a Contribuição do Hospital para a Preservação do Meio Ambiente. Revista Academia de Enfermagem, 2(2):21-29. 
Fachin, O. (2001). Fundamentos de metodologias. Editora Atlas.

Faria, R. A. (2007). Resíduos perfurocortantes gerados em um hospital materno infantil de Goiânia: gerenciamento e riscos ocupacionais. Goiânia: Faculdade de Engenharia/UFG.

Ferreira, J. A. \& Anjos, L. A. dos. (2001). Aspectos de saúde coletiva e ocupacional associados à gestão dos resíduos sólidos municipais. Cadernos de saúde Pública, 17(3):689-696.

Ferreira, J. A. (1997). Lixo hospitalar e domiciliar: semelhanças e diferenças: estudo de caso no município do Rio de Janeiro. Rio de Janeiro: Fundação Oswaldo Cruz.

Filho, V. O. R. (2015). Seminário Hospitais Saudáveis, in: II Fórum Vigilância Sanitária de Resíduos de Serviço de Saúde.

Mendonça, I. V. S., Oliveira, L. P. \& Caldas, A.J.M. (2019). Acidentes de trabalho entre profissionais da limpeza hospitalar em uma capital do Nordeste, Brasil. Ciência e Saúde Coletiva, 24(11):4123-4132.

Leite, V. D. \& Lopes, W. S. (2000). Avaliação dos aspectos sociais, econômicos e ambientais causados pelo lixão da cidade de Campina Grande, In: IX Simpósio Luso-Brasileiro de Engenharia Sanitária e Ambiental, Anais, CD-ROM IV, Porto Seguro, Associação Brasileira de Engenharia Sanitária e Ambiental.

Maglio, I. C. (2000). Gestão ambiental dos resíduos sólidos - o papel dos Municípios. Anais do $9^{\circ}$ Simpósio Luso-Brasileiro de Engenharia Sanitária e Ambiental, Porto Seguro, Associação Brasileira de Engenharia Sanitária e Ambiental.

Moura, G. M. S. S. \& Doi, K. M. (2011). Resíduos sólidos de serviços de saúde: uma fotografia do comprometimento da equipe de enfermagem. Revista Gaúcha de Enfermagem, 32(2):338-344.

Mol, M. P. G. (2016). Risco de infecção pelos vírus das hepatites B e C nos trabalhadores da coleta de resíduos de serviços de saúde em Belo Horizonte $M G$. Minas Gerais: Universidade Federal de Minas Gerais.

Oliveira, L. L., Souza, P. M., Clementino, F. S..; Almeida, J. L. S., Cardoso, N. V. \& Carvalho, L. M. A. (2021) Resíduos de serviços de saúde: a responsabilidade ambiental na visão do profissional da Atenção Básica. Pesquisa, Sociedade e Desenvolvimento, 10(3):e28610313206, 2021.

Robazzi, M. L.C. C., Moriya, T. M., Fávero, M. \& Pinto, P. H. D. (1992). Algumas considerações sobre o trabalho dos coletores de lixo. Revista Brasileira de Saúde Ocupacional, 20(76):34-41.

Rocha, J. V. R, Rocha, L.S. D. S. \&Madureira, M. T. (2021). A importância do tratamento adequado e descarte de resíduos de saúde em tempos de pandemia de Covid-19. Pesquisa, Sociedade e Desenvolvimento, Research, society and Development,10(15): e260101522807.

Sanches, P. S. (1995) Caracterização dos Riscos nos Resíduos de Serviço de Saúde e na Comunidade. In: Gerenciamento de Resíduos Sólidos de Serviço de Saúde. São Paulo: CETESB.

Secretaria Especial de Previdência e Trabalho (1978). Norma Regulamentadora $n^{o}$ 9. Programa de Prevenção de Riscos Ambientais. https://www.gov.br/trabalho-e-previdencia/pt-br/composicao/orgaos-especificos/secretaria-de-trabalho/inspecao/seguranca-e-saude-no-trabalho/ctppnrs/norma-regulamentadora-no-9-nr-9

Silva, D. F., Sperling, E. V. \& Barros, R. T. V. (2014). Avaliação do gerenciamento dos resíduos de serviços de saúde em municípios da região metropolitana de Belo Horizonte (Brasil). Engenharia Sanitária e Ambiental, 19(3):251-262.

Silva, A. S., Silva, J. G., Moreira, F. N. C., Santos, M. L. S. \& Mourão, F. V. (2020). Analysis of health service waste management in a public hospital inthe municipality of Paragominas. Research, Society and Development. 9(7):1-23.

Sinoti, A. L. L. (2009). Gerenciamento de Resíduos de Serviços de Saúde: Conceitos e análise de risco.

Uehara, S. C. S. A., Veiga, T. B. \& Takayanagui, A. M. M. ( 2019). Gerenciamento de resíduos de serviços de saúde em hospitais de Ribeirão Preto (SP), Brasil. Engenharia Sanitária e Ambiental, 24(1):121-130.

Velloso, M. P.; Santos, E. M.; Anjos, L. A. (1997). Processo de trabalho e acidentes de trabalho em coletores de lixo domiciliar na cidade do Rio de Janeiro, Brasil. Cadernos de Saúde Pública, 13(4):693-700.

World Health Organization (2014). Safe management of wastes from health-care activities. Geneva:WHO.

World Organization Health (1999). Safe management of wastes from health care activities. Geneva: WHO.

Zepeda, F. (1995). El Manejo de Residuos Sólidos Municipales En América Latina y El Caribe. Washington: Organización Panamericana de la Salud. 